\title{
An Analysis of the Prognostic Factors Affecing the Clinical Outcomes of Conventional Lumbar Open Discectomy : Clinical and Radiological Prognostic Factors
}

\author{
Jae Chul Lee, Min-Soo Kim, Byung-Joon Shin \\ Department of Orthopaedic Surgery \& Spine Center, Soonchunhyang University College of Medicine, Seoul, Korea
}

Study Design: This is a retrospective study.

Purpose: We wanted to examine the clinical and radiological prognostic factors affecting the postoperative clinical outcome of patients with lumbar disc herniation and who underwent open discectomy.

Overview of Literature: Conventional open discectomy has been widely used as a treatment regimen for the management of lumbar disc herniation. Still, much controversy exists regarding the factors that affect the postoperative clinical outcomes.

Methods: The current study was conducted on 40 patients who were diagnosed with lumbar disc herniation by the senior surgeon of our department from March 2004 to June 2007. These patients were refractory to conservative treatment and they could be followed up for more than one year following their surgical treatments. Preoperatively, after postoperative year 1 and at the final follow-up, a comparison was made for the Oswestry disability index (ODI) scores and the visual analogue scale (VAS) scores that indicated low back pain and radiating pain. For identifying prognostic factors, an analysis was also performed for such factors as age, gender, the operated level, the duration of preoperative low back pain and radiating pain, a smoking history, the body mass index and whether the surgery was revision or the primary operation. A radiological analysis, based on the preoperative plain flexion-extension radiography, was performed for the presence of mild segmental instability of $3 \mathrm{~mm}$, spondylolysis and disc space narrowing. Pfirrmann's degenerative grade of the disc, the degree of herniation and whether a herniation was central or massive on the magnetic resonance imaging scans.

Results: At the final follow-up, the ODI was significantly higher in the cases of revision as compared with the cases of primary operation. The female gender also had a tendency for a poor ODI as compared with that of the men, but this had only borderline statistical significance. There was significant correlation between the preoperative ODI and the preoperative VAS indicating radiating pain. At a final follow up, the low back pain VAS score was significantly lower in the extruded cases as compared with that of the protruded or sequestrated cases.

Conclusions: Following an analysis for detecting the prognostic factors of open discectomy, the final clinical outcome was found to be poor for the revision surgery cases. In regard to the type of herniation, the degree of low back pain was relatively lower at a final follow-up for the extruded cases as compared with that for the protruded or sequestrated cases. Open discectomy surgery should be performed after evaluating the patients' various prognostic factors that could affect the final clinical outcome.

Key Words: Lumbar disc herniation, Open discectomy, Clinical outcome, Prognostic factor

Received Sep 23, 2009; 1st Revised Dec 14, 2009; Accepted Dec 16, 2009

Corresponding author: Byung-Joon Shin, MD

Department of Orthopaedic Surgery \& Spine center, Soonchunhyang University College of Medicine,

657 Hannam-dong, Yongsan-gu, Seoul 140-743, Korea

Tel: +82-2-709-9051, Fax: +82-2-796-3682, E-mail: schsbj@ @osp.sch.ac.kr 


\section{Introduction}

Disc herniation is one of the most common spinal diseases, and lumbar open discectomy is the most frequently used methods for treating lumbar disc hernation that requires spinal surgery [1]. Lumbar open discectomy was first introduced by Mixter and Barr [2] in 1934. Since then, it has been widely used as the basic surgical regimen for the treatment of disc herniation. Discectomy has been performed in recent years by using an endoscope, but conventional open discectomy's effectiveness has been definitely confirmed for the treatment of lumbar disc herniation. Open discectomy has been shown to produce good treatment outcomes in 70-90\% of the cases [3-6]. It is assumed that preoperatively, accurately determining the indications for this surgery is essential for producing good treatment outcomes. Determining the prognostic factors that affect the surgical outcomes would be helpful for predicting the surgical outcomes and selecting the optimal treatment modality. Magnetic resonance imaging (MRI) has excellent sensitivity and specificity to diagnose disc herniation and it can be used to assess the severity and level of disc herniation. Further, MRI can assess the internal change of discs from a radiological perspective.

Numerous studies have previously been conducted to examine the factors affecting the surgical treatment outcomes of disc herniation. However, to the best of our knowledge, few studies have been conducted on the treatment outcomes of disc herniation using such technicallyadvanced imaging modalities as 1.5-T MRI equipment and with assessing the clinical outcomes by having the patients use a subjective pain score visual analogue scale (VAS) or using the Oswestry disability index (ODI).

Given the above background, we analyzed the correlations between the clinical, radiological prognostic factors and postoperative clinical outcomes of patients who underwent open discectomy under the diagnosis of lumbar disc herniation.

\section{Materials and Methods}

\section{Study subjects}

The current study was conducted on 40 patients who were diagnosed as having lumbar disc herniation and who were operated on by the senior surgeon of our department from March 2004 to June 2007. These patients were refractory to conservative treatment and they could be followed up for more than one year following their surgical treatments. The patients with worker's compensation and those who were involved with motor vehicle accidents were excluded from the current analysis.

There were 23 men (58\%) and 17 women (43\%). The total patients ranged in age from 15 to 83 years and the males' mean age was 34 years and the females' mean age was 50. The duration of follow up was 23.5 months (range, 12 to 49 months). In regard to the operated level, there were 23 cases of L4-5, nine cases of L5-S1, five cases of L3-4 and three cases of L2-3. There were eight smokers and 32 non-smokers.

\section{Study methods}

All the surgery was performed by a senior orthopedic spine surgeon. The indications for surgery included the following: treatment failure after conservative treatment for 6 weeks, the presence of motor weakness without improvement or progressive neurologic deficit, and patients for which their daily lives could not be maintained due to the frequent occurrence of lower extremity radiating pain.

The next day after surgery, the patients were recommended to wear a corset and they were encouraged to ambulate. A length of the hospitalization period was usually shorter than one week.

\section{(1) An analysis of the clinical factors and outcomes}

At our department, recording of the preoperative clinical condition was done using a protocol for the patients who underwent surgery for the management of disc herniation. We compared the preoperative, postoperative year 1 and final follow-up ODI scores and the VAS scores, which indicated lower back pain (LBP) and radiating pain. To identify the prognostic factors, an analysis was performed for such factors as age, gender, the surgical sites (levels), the duration of preoperative low back pain and radiating pain, a smoking history, the body mass index (BMI) and whether a surgery was a revision operation or the primary operation.

For the assessment of the postoperative clinical outcomes, the ODI and VAS scores for low back pain and radiating pain at a postoperative final follow-up were used. The amount of the reduction of the final follow-up ODI scores, as compared to the preoperative ODI, was also used. 
(2) The radiological prognostic factors

The presence of mild segmental instability of $<3 \mathrm{~mm}$, spondylolysis and disc space narrowing were assessed on the radiological analysis based on the preoperative plain flexion-extension radiography. Disc space narrowing was defined as a decrease of the relative disc height by more than $30 \%$ as compared with the height of the upper disc. On MRI scans, the degree of disc herniation was classified as the protruded type, the extruded type and the sequestrated type according to the criteria of Costello and Beall [7] and a 5 -scale degenerative grade of the disc proposed by Pfirrmann et al. [8]. We also assessed whether there was central herniation or massive herniation of the intervertebral disc.

\section{(3) Statistical analysis}

The scores indicating the clinical outcomes postoperatively and at a final follow-up were used for univariate analysis. Mann-Whitney U tests and Kruskal-Wallis tests were used for the analysis of statistical significance depending on the presence of various factors. Pearson correlation analysis was used for the assessment of correlation between the continuous variables and the scores indicating the clinical outcomes at a final follow-up. Multiple logistic regression analysis was performed for concurrent consideration of multiple factors. $p$-values $<0.05$ were considered statistically significant.

\section{Results}

\section{Changes in the clinical parameters before and after open discectomy}

The duration of preoperative low back pain (mean \pm standard deviation) was $66 \pm 103$ weeks and that of radiating pain was $9 \pm 22$ weeks. The mean low back pain VAS was $3.8 \pm 3.0$ and the mean radiating pain VAS was $7.5 \pm$ 1.5. The mean preoperative ODI was $27 \pm 8$. At the final

Table 1. Changes of clinical parameters

\begin{tabular}{lccc}
\hline \hline & Preoperative & Final follow-up & $p$-value \\
\hline LBP VAS score & $3.8 \pm 3.0$ & $1.2 \pm 1.5$ & 0.000 \\
RP VAS score & $7.5 \pm 1.5$ & $0.2 \pm 0.5$ & 0.000 \\
ODI & $27 \pm 8.0$ & $5.5 \pm 5.8$ & 0.000 \\
\hline
\end{tabular}

Values are presented as mean \pm standard deviation.

LBP: Low back pain, RP: Radiating pain, VAS: Visual analogue scale, ODI: Oswestry disability index. follow-up, the mean low back pain VAS was $1.2 \pm 1.5$, the mean radiating pain VAS was $0.2 \pm 0.5$ and the mean ODI was $5.5 \pm 5.8$. These parameters were all significantly decreased as compared to the preoperative values $(p=$ 0.000) (Table 1).

\section{Results of the radiological analysis}

An analysis of the radiological findings was performed by two orthopaedic surgeons. For the case with an inconsistent reading, a consensus reading was done by the orthopaedic surgeons. Radiologically, of the 40 cases, there was one case in which mild segmental instability of less than $3 \mathrm{~mm}$ was present. Disc space narrowing was present in 22 cases

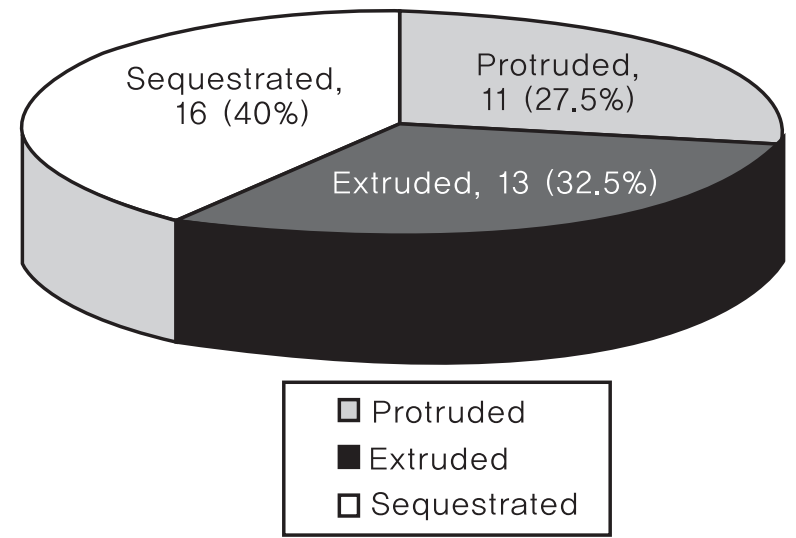

Fig. 1. Herniation type.

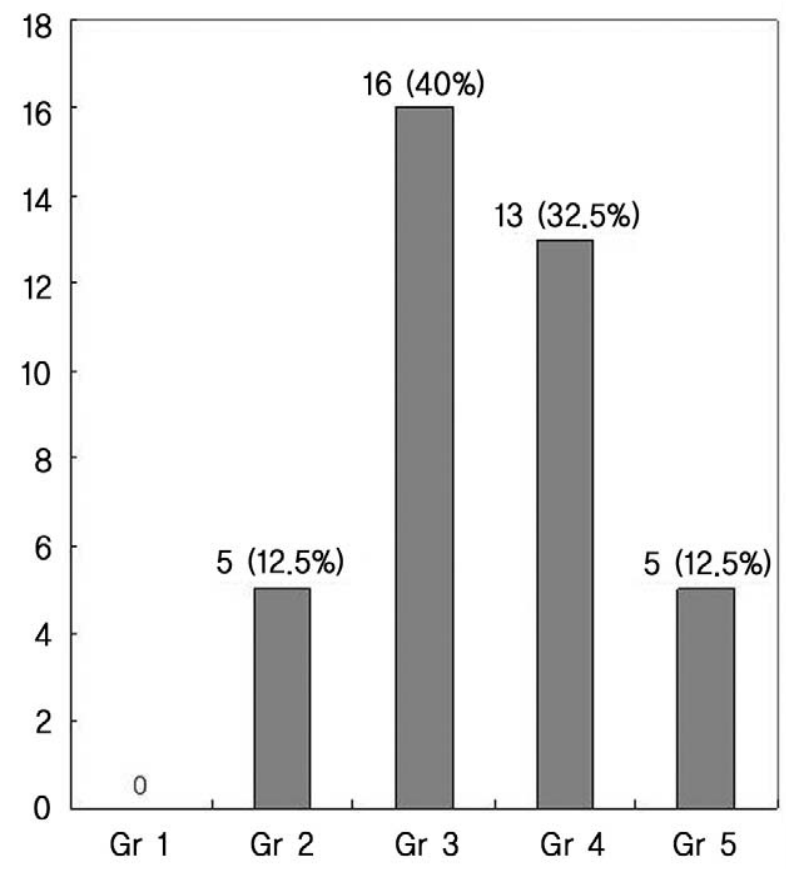

Fig. 2. According to Pfirrmann classification. 
$(55 \%)$ and spondylolysis was present in four cases $(10 \%)$. In regard to the type of disc herniation, there were 11 cases of protruded herniation, 13 cases of extruded herniation and 16 cases of sequestrated herniation (Fig. 1). There were eight cases $(20 \%)$ of central herniation and nine cases (22.5\%) of massive herniation. According to the Pfirrmann classification of disc degeneration, there were five cases of grade 5, 13 cases of grade 4, 16 cases of grade 3, five cases of grade 2 and 0 cases of grade 1 (Fig. 2).

\section{Analysis of the factors affecting ODI at a final fol- low-up}

At a final follow up, the ODI was significantly higher in

Table 2. Analysis of prognostic factors for Oswestry disability index at final follow-up

\begin{tabular}{|c|c|c|c|}
\hline & $\mathrm{n}(\%)$ & Final ODI & $p$-value \\
\hline Sex & & & 0.086 \\
\hline Male & $23(57.5)$ & $4.2 \pm 3.9$ & \\
\hline Female & $17(42.5)$ & $7.4 \pm 7.4$ & \\
\hline Smoking & & & 0.099 \\
\hline Yes & $8(20.0)$ & $2.5 \pm 2.2$ & \\
\hline No & $32(80.0)$ & $6.3 \pm 6.2$ & \\
\hline Level & & & 0.541 \\
\hline L2-3 & $3(7.5)$ & $2.7 \pm 4.6$ & \\
\hline L3-4 & $5(12.5)$ & $3.6 \pm 4.2$ & \\
\hline L4-5 & $9(22.5)$ & $6.6 \pm 6.7$ & \\
\hline L5-S1 & $23(57.5)$ & $4.8 \pm 4.3$ & \\
\hline Primary or Revision & & & $0.043^{\mathrm{a})}$ \\
\hline Primary surgery & $32(80.0)$ & $4.0 \pm 3.6$ & \\
\hline Revision surgery & $8(20.0)$ & $10.9 \pm 8.6$ & \\
\hline Minimal segmental instability & & & 0.341 \\
\hline Yes & $1(2.5)$ & 0 & \\
\hline No & $39(97.5)$ & $5.7 \pm 5.8$ & \\
\hline Disc space narrowing & & & 0.192 \\
\hline Yes & $22(55.0)$ & $6.6 \pm 7.3$ & \\
\hline No & $18(45.0)$ & $4.3 \pm 2.9$ & \\
\hline Spondylolysis & & & 0.277 \\
\hline Yes & $4(10.0)$ & $2.5 \pm 2.9$ & \\
\hline No & $36(90.0)$ & $5.9 \pm 6.0$ & \\
\hline Herniation type & & & 0.403 \\
\hline Protruded & $11(27.5)$ & $5.3 \pm 3.0$ & \\
\hline Extruded & $13(32.5)$ & $4.0 \pm 7.0$ & \\
\hline Sequestrated & $16(40.0)$ & $6.9 \pm 6.1$ & \\
\hline Pfirrmann grade & & & 0.539 \\
\hline Grade I & $0(0.0)$ & & \\
\hline Grade II & $5(12.5)$ & $3.4 \pm 3.7$ & \\
\hline Grade III & $16(40.0)$ & $5.1 \pm 4.0$ & \\
\hline Grade IV & $13(32.5)$ & $6.1 \pm 6.3$ & \\
\hline Grade V & $5(12.5)$ & $8.6 \pm 10.4$ & \\
\hline Central herniation & & & 0.779 \\
\hline Yes & $8(20.0)$ & $6.1 \pm 7.6$ & \\
\hline No & $32(80.0)$ & $5.5 \pm 5.1$ & \\
\hline Massive herniation & & & 0.264 \\
\hline Yes & $9(22.5)$ & $7.4 \pm 8.4$ & \\
\hline No & $31(77.5)$ & $5.0 \pm 4.8$ & \\
\hline
\end{tabular}

Values are presented as mean \pm standard deviation.

ODI: Oswestry disability index.

a) $p<0.05$ 
the revision cases $(10.9 \pm 8.6)$ as compared with that of the primary operation cases $(4.0 \pm 3.6)(p=0.043)$. The female gender showed a tendency $(p=0.086)$ for a higher ODI (7.4 \pm 7.4$)$ as compared with that of the men $(4.2 \pm$ 3.9). However, the ODI scores at a final follow-up did not show a significant difference depending on such factors as a smoking history, the levels of herniation, mild segmental instability, disc space narrowing, Pfirrmann's degenerative grade, the type of herniation, central herniation or massive herniation and the presence of spondylolysis (Table 2).

In addition, age, BMI, smoking, the duration of preoperative low back pain or radiating pain and the VAS scores of the preoperative low back pain or radiating pain had no significant correlations with a final follow-up ODI score (Table 3).

The multiple regression analysis to identify the factors affecting whether the ODI scores were lower or higher than 10 points at a final follow-up showed statistical significance for whether surgery was revision or the primary operation. The revision cases, as compared with the cases of primary

Table 3. Correlation of prognostic factors with final Oswestry disability score

\begin{tabular}{lc}
\hline \hline & $p$-value \\
\hline Age & 0.269 \\
BMI & 0.819 \\
Preop. LBP VAS & 0.613 \\
Preop. RP VAS & 0.175 \\
LBP duration & 0.881 \\
RP duration & 0.710 \\
\hline
\end{tabular}

BMI: Body mass index, Preop.: Preoperative, LBP: Low back pain, RP: Radiating pain, VAS: Visual analogue scale. surgery, were 36.4 times more likely to have the ODI score more than 10 points at a final follow-up (Table 4).

\section{Analysis of the factors affecting the amount of reduction of the ODI scores}

On the analysis of the statistical significance between the amount of reduction of the ODI scores and various prognostic factors, there was a significant correlation of the amount of reduction of the ODI scores with the preoperative VAS scores of lower extremity radiating pain ( $p=0.003$, determinant coefficient $\mathrm{R}^{2}=0.208$ ).

\section{Analysis of the factors affecting low back pain VAS at a final follow-up}

At a final follow up, the degree of low back pain VAS was significantly lower in the extruded cases $(0.2 \pm 0.6)$, as compared with that of the protruded $(1.6 \pm 1.4)$ or sequestrated cases $(1.6 \pm 1.7)(p=0.010)$. However, according to the factors such as gender, smoking, the level of herniation, mild segmental instability, disc space narrowing, Pfirmann's degeneration grade and the presence of central herniation, massive herniation and spondylolysis, there was no significant difference in the LBP scores at a final follow-up (Table 5).

In addition, at a final follow-up, the following factors were found to have no significant correlation with the final LBP scores: age, BMI, smoking, the duration of preoperative LBP and radiating pain, and the VAS scores of the preoperative low back pain and the radiating pain (Table 6).

Table 4. Logistic regression analysis of prognostic factors to final Oswestry disability index $(>10$ or $<10)$

\begin{tabular}{|c|c|c|c|c|}
\hline \multirow{2}{*}{ Risk factor } & \multirow{2}{*}{$p$-value } & \multirow{2}{*}{$\operatorname{Exp}(B)$} & \multicolumn{2}{|c|}{$95 \%$ confidence interval } \\
\hline & & & Lower & Upper \\
\hline Age & 0.791 & 1.016 & 0.905 & 1.139 \\
\hline Sex (female) & 0.879 & 1.318 & 0.037 & 46.817 \\
\hline Revision (vs. Primary) & $0.017^{\mathrm{a})}$ & 36.454 & 1.927 & 689.57 \\
\hline Preop. LBP VAS & 0.894 & 0.972 & 0.642 & 1.472 \\
\hline Preop. RP VAS & 0.349 & 0.523 & 0.135 & 2.028 \\
\hline Preop. Oswestry disability index & 0.582 & 0.942 & 0.762 & 1.165 \\
\hline Type & 0.432 & & & \\
\hline Extruded (vs. Protruded) & 0.627 & 2.868 & 0.041 & 201.257 \\
\hline Sequestrated (vs. Protruded) & 0.232 & 10.527 & 0.221 & 501.463 \\
\hline
\end{tabular}

Preop.: Preoperative, LBP: Low back pain, RP: Radiating pain, VAS: Visual analogue scale. a) $p<0.05$. 
Table 5. Analysis of prognostic factors for low back pain score at final follow-up

\begin{tabular}{|c|c|c|c|}
\hline & $\mathrm{n}(\%)$ & Final LBP VAS & $p$-value \\
\hline Sex & & & 0.467 \\
\hline Male & $23(57.5)$ & $1.0 \pm 1.5$ & \\
\hline Female & $17(42.5)$ & $1.4 \pm 1.5$ & \\
\hline Smoking & & & 0.053 \\
\hline Yes & $8(20.0)$ & $0.38 \pm 1.0$ & \\
\hline No & $32(80.0)$ & $1.3 \pm 1.5$ & \\
\hline Level & & & 0.189 \\
\hline L2-3 & $3(7.5)$ & $1.0 \pm 1.7$ & \\
\hline L3-4 & $5(12.5)$ & $1.6 \pm 1.7$ & \\
\hline L4-5 & $9(22.5)$ & $1.4 \pm 1.6$ & \\
\hline L5-S1 & $23(57.5)$ & $0.2 \pm 0.7$ & \\
\hline Primary or Revision & & & 0.872 \\
\hline Primary surgery & $32(80.0)$ & $4.0 \pm 3.6$ & \\
\hline Revision surgery & $8(20.0)$ & $10.9 \pm 8.6$ & \\
\hline Minimal segmental instability & & & 0.443 \\
\hline Yes & $1(2.5)$ & & \\
\hline No & $39(97.5)$ & $1.2 \pm 1.5$ & \\
\hline Disc space narrowing & & & 0.265 \\
\hline Yes & $22(55.0)$ & $0.9 \pm 1.3$ & \\
\hline No & $18(45.0)$ & $1.4 \pm 1.7$ & \\
\hline Spondylolysis & & & 0.105 \\
\hline Yes & $4(10.0)$ & $0.25 \pm 0.5$ & \\
\hline No & $36(90.0)$ & $1.3 \pm 1.5$ & \\
\hline Herniation type & & & $0.010^{\mathrm{a})}$ \\
\hline Protruded & $11(27.5)$ & $1.6 \pm 1.4$ & \\
\hline Extruded $^{\mathrm{b})}$ & $13(32.5)$ & $0.2 \pm 0.6$ & \\
\hline Sequestrated & $16(40.0)$ & $1.6 \pm 1.7$ & \\
\hline Pfirrmann grade & & & 0.625 \\
\hline Grade I & $0(0.0)$ & & \\
\hline Grade II & $5(12.5)$ & $1.6 \pm 1.5$ & \\
\hline Grade III & $16(40.0)$ & $1.2 \pm 1.7$ & \\
\hline Grade IV & $13(32.5)$ & $1.3 \pm 1.4$ & \\
\hline Grade V & $5(12.5)$ & $0.4 \pm 0.9$ & \\
\hline Central herniation & & & 0.646 \\
\hline Yes & $8(20.0)$ & $1.0 \pm 1.8$ & \\
\hline No & $32(80.0)$ & $1.3 \pm 1.4$ & \\
\hline Massive herniation & & & 0.244 \\
\hline Yes & $9(22.5)$ & $1.7 \pm 1.8$ & \\
\hline No & $31(77.5)$ & $1.0 \pm 1.4$ & \\
\hline
\end{tabular}

LBP: Low back pain, VAS: Visual analogue scale.

${ }^{\text {a) }} p<0.05$ by ANOVA.

${ }^{b}$ Significant difference from other 2 groups (protruded and sequestrated) by post hoc test of Tukey.

\section{Analysis of the factors affecting radiating pain VAS at a final follow-up}

At a final follow-up and based on statistical analysis, there were no preoperative clinical factors or radiological factors affecting the radiating pain VAS scores.

\section{Discussion}

Numerous studies have reported that the treatment outcomes were satisfactory in approximately more than $80 \%$ of patients who underwent conventional open discectomy [36]. To obtain good postoperative treatment outcomes in 
Table 6. Correlation of prognostic factors with low back pain score at final follow-up

\begin{tabular}{lc}
\hline \hline & $p$-value \\
\hline Age & 0.116 \\
BMI & 0.764 \\
Preop. LBP VAS & 0.721 \\
Preop. RP VAS & 0.434 \\
LBP duration & 0.445 \\
RP duration & 0.735 \\
\hline
\end{tabular}

BMI: Body mass index, Preop.: Preoperative, LBP: Low back pain, RP: Radiating pain, VAS: Visual analogue scale.

patients with lumbar disc herniation, an adequate evaluation of the prognostic factors is mandatory with the accurate determination of the surgical indications, and many studies have already analyzed various prognostic factors. These studies have identified several significant prognostic factors. Yet in recent years, according to the newer indices for clinical outcomes such as the VAS or ODI, the quantification and objectification of clinical outcomes have become possible in a manner that is different from the previous clinical assessment methods performed by physicians and as rated on a 4-grade scale. With the widespread use of a 1.5-T MRI, high-quality images can be obtained for the assessment of the severity and type of disc herniation and disc degeneration. According to these facts, our study has made progress from previous study. Thus, it is expected that various prognostic factors can be analyzed more accurately. With methods used in current study. Given this background, attempts have been made to analyze the clinical and radiological prognostic factors using not only the clinical outcome parameters that can be better quantified and then could be used to predict the treatment outcomes following discectomy, but also technically-advanced MRI equipment.

To date, many studies have been conducted to examine the relationship between the treatment outcomes of conventional open discectomy and the age of patients. According to Hurme and Alaranta [9], the treatment outcomes were found to be poor in patients aged 40 years or older at a 6 month postoperative follow-up in 176 patients who were surgically treated. Weir [5] and Weber [6] also noted that the treatment outcomes were poor at follow-up as the patient age increased. Yet according to Long et al. [3] and Herron and Turner [10], there was no significant correlation between age and the postoperative outcomes. In our series, there was also no significant correlation between age and the postoperative outcomes. This implies that open discec- tomy rather than spinal fusion could be considered as the primary surgery for disc herniation in the elderly patients who have no concurrent presence of severe stenosis or degenerative lesion.

Many studies have examined an influence of gender on the result of discectomy. According to Manniche et al. [4], the surgical treatment outcomes of 261 patients were poorer for the female patients as compared with their male counterparts. Kosteljanetz et al. [11] also noted that the surgical treatment outcomes were poorer in female patients as compared with their male counterparts. Weber [6] analyzed the treatment outcomes depending on gender in 280 patients with lumbar disc herniation. According to that study, the treatment outcomes were better in the male patients as compared with their female counterparts, but there was no statistically significant difference in the treatment outcomes between the two groups. Also in our series, the final ODI was slightly higher for the female patients as compared with their male counterparts, yet only borderline statistical significance was found. Further larger-scale studies on this subject are needed in the future.

It has been reported that postoperative low back pain is significantly correlated with patients who have greater than a 15-pack-year smoking history [12]. Yet in our series, there was no significant correlation between the postoperative low back pain score and a smoking history. It is assumed that smoking has no great impact because multiple factors are involved in postoperative low back pain. In the current study, because the smoking period and amount of smoking were not considered, unlike the above studies, it would be problematic to draw a hasty conclusion. This also deserves further study.

A longer duration of radiating pain has been reported to have a relationship with poor treatment outcomes according to Hurme and Alaranta [9] and Jonsson [13]. But according to Manniche et al. [4], there were no significant differences in the preoperative duration of symptom and the postoperative results. Also in our series, the duration of radiating pain had no effects on a final follow-up ODI and the low back pain and radiating pain scores. These results indicate that neurologic recovery can be expected after discectomy even in the patients who have long standing radiculopathy due to disc herniation.

As for the operated level, Weir reported that L5-S1 lesion had better clinical outcomes for reducing postoperative low back pain and radiating pain as compared with L4-5 lesion [5], but there are many studies reporting that there was no 
significant difference depending on the affected level. In association with this, Manniche et al. [4] and Kim et al. [14] reported that there was no significant difference depending on the level. In our series, a comparison was differentially made for such levels as L2-3, L3-4, L4-5 and L5-S1. This showed that there was no significant difference in the final clinical outcome depending on the affected segment.

According to Dabbs and Dabbs [15], there was no significant correlation between the preoperative disc space narrowing and post-discectomy low back pain. Nah et al. [16] also reported that there was no significant correlation between the disc space narrowing and the postoperative clinical outcome. In our series, there were no significant correlations between disc space narrowing and the postoperative low back pain and clinical outcomes. Accordingly, these results indicate that it is not necessary to hastily consider performing spinal fusion even for cases in which there are radiological degenerative changes such as disc space narrowing.

According to the studies where a comparative analysis was performed for revision cases and the primary operation, Papadopoulos et al. [17] reported that there were no significant correlations between the severity of low back pain and radiating pain and the severity of numbness and a tingling sensation of the lower extremity and all the functional outcomes. Cinotti et al. [18] compared 26 patients who underwent revision surgery with those who underwent a primary operation. These authors noted that there were no significant differences in the clinical outcomes. However, according to a number of studies that focused on revision discectomy, the presence of fibrosis in the operative field was associated with poor treatment outcomes for patient suffering from recurrence of radiating pain. This implies that the fibrous tissue surrounding the dura and nerve roots might cause disturbance and delay of neural recovery. It is also presumed that the additional removal of disc and the resulting disc injury might contribute to the occurrence of early disc degeneration. In the current study, the clinical outcomes were significantly poorer in the revision cases as compared with those of the primary surgery cases. This indicates that patients should be given sufficient explanation about the clinical outcomes in cases in which a revision surgery is performed and the patients' written informed consent should be obtained.

According to Manniche et al. [4], who classified the type of disc herniation into the normal disc, the extruded type and the sequestrated one using myelography and then they compared the postoperative treatment outcomes, the treatment outcomes were found to be good in the sequestrated and extruded cases. Herron and Turner [10] reported that the treatment outcomes were better in cases in which the disc hernation was seen to be placed lateral to the spinal canal on the myelography findings as compared with those in which the disc hernation was placed central to the spinal canal. In our series, we comparatively analyzed the clinical outcomes based on the protruded, extruded and sequestrated classification of disc herniation with using 1.5-T MRI, and the resolution of 1.5-T MRI is markedly improved from the conventional types of myelography. Our results showed that the low back pain VAS score was significantly lower at a final follow-up for the extruded cases as compared with that of the other cases. But there was no significant difference of the ODI score depending on the herniation type. This might be because the differences were difficult to detect because the ODI score improvement was so large for all the types of herniation due to improvement of the radiating pain.

Yorimitsu et al. [19] reported that the occurrence of preoperative disc degeneration was significantly associated with postoperative low back pain, but our results showed that the severity of disc degeneration, which was classified into five grades based on MRI scans, had no significant effect on the clinical outcomes following surgical treatment. This was in agreement with the report by Kim et al. [14], who stated that performing spinal fusion cannot be considered solely based on the degenerative change seen on MRI scans, and the severity of degeneration was not proportional to the final clinical outcomes.

\section{Conclusions}

On the analysis of the prognostic factors for conventional open discectomy, the final clinical outcomes were found to be poor in the revision cases. In regard to the types of disc herniation, the degree of residual low back pain was significantly lower at a final follow-up in the extruded cases as compared with that of the protruded or sequestrated cases.

For patients who are to undergo open discectomy, surgery should be performed with seriously considering the various prognostic factors that affect the clinical outcomes. 


\section{REFERENCES}

1. Kohlboeck G, Greimel KV, Piotrowski WP, et al. Prognosis of multifactorial outcome in lumbar discectomy: a prospective longitudinal study investigating patients with disc prolapse. Clin J Pain 2004;20:455-61.

2. Mixter WJ, Barr JS. Rupture of the intervertebral disc with involvement of the spinal canal. New Engl J Med 1934; 211:210-4.

3. Long CJ, Brown DA, Engelberg J. Intervertebral disc surgery: strategies for patient selection to improve surgical outcome. J Neurosurg 1980;52:818-24.

4. Manniche C, Asmussen KH, Vinterberg H, Rose-Hansen EB, Kramhoft J, Jordan A. Analysis of preoperative prognostic factors in first-time surgery for lumbar disc herniation, including Finneson's and modified Spengler' s score systems. Dan Med Bull 1994;41:110-5.

5. Weir BK. Prospective study of 100 lumbosacral discectomies. J Neurosurg 1979;50:283-9.

6. Weber H. Lumbar disc herniation: a controlled, prospective study with ten years of observation. Spine (Phila Pa 1976) 1983;8:131-40.

7. Costello RF, Beall DP. Nomenclature and standard reporting terminology of intervertebral disk herniation. Magn Reson Imaging Clin N Am 2007;15:167-174.

8. Pfirrmann CW, Metzdorf A, Zanetti M, Hodler J, Boos N. Magnetic resonance classification of lumbar intervertebral disc degeneration. Spine (Phila Pa 1976) 2001;26:1873-8.

9. Hurme M, Alaranta H. Factors predicting the result of surgery for lumbar intervertebral disc herniation. Spine (Phila Pa 1976) 1987;12:933-8.

10. Herron LD, Turner J. Patient selection for lumbar laminectomy and discectomy with a revised objective rating sys- tem. Clin Orthop Relat Res 1985;199:145-52.

11. Kosteljanetz M, Espersen JO, Halaburt H, Miletic T. Predictive value of clinical and surgical findings in patients with lumbago-sciatica: a prospective study (part I). Acta Neurochir (Wien) 1984;73:67-76.

12. Hanley EN Jr, Shapiro DE. The development of low-back pain after excision of a lumbar disc. J Bone Joint Surg Am 1989;71:719-21.

13. Jonsson B. Patient-related factors predicting the outcome of decompressive surgery. Acta Orthop Scand Suppl 1993;25 1:69-70.

14. Kim EH, Woo BC, Cho DY. Prognostic factors after conventional surgery of lumbar disc herniation: comparative study between non-compensator and compensator. J Korean Soc Spine Surg 1997;4:18-26.

15. Dabbs VM, Dabbs LG. Correlation between disc height narrowing and low-back pain. Spine (Phila Pa 1976) 1990;15:1366-9.

16. Nah HY, Kim YT, Ahn HS, Kim KY. Lumbar intervertebral disc: a histologic, radiologic and clinical correlations based on over 95 discectomies. J Korean Soc Spine Surg 1994;1:66-73.

17. Papadopoulos EC, Girardi FP, Sandhu HS, et al. Outcome of revision discectomies following recurrent lumbar disc herniation. Spine (Phila Pa 1976) 2006;31:1473-6.

18. Cinotti G, Roysam GS, Eisenstein SM, Postacchini F. Ipsilateral recurrent lumbar disc herniation: a prospective, controlled study. J Bone Joint Surg Br 1998;80:825-32.

19. Yorimitsu E, Chiba K, Toyama Y, Hirabayashi K. Longterm outcomes of standard discectomy for lumbar disc hermiation: a follow-up study of more than 10 years. Spine (Phila Pa 1976) 2001;26:652-7. 